\title{
Performance and Stability Analysis of a Message Oriented Reliable Multicast for Distributed Virtual Environments in Java
}

\author{
Gunther Stuer, Jan Broeckhove, and Frans Arickx \\ Antwerp University \\ Department of Mathematics and Computer Sciences \\ Groenenborgerlaan 171, 2020 Antwerp, Belgium \\ gstuer@ruca.ua.ac.be
}

\begin{abstract}
The aim of this paper is to present the performance and stability analysis of a reliable multicast system. It has been optimized for use in distributed virtual environments and is implemented in Java. The paper will describe the characteristics of our reliable multicast implementation, as observed in our test environment. We will also compare with non-reliable multicast protocols.
\end{abstract}

\section{Introduction}

This paper describes the performance analysis of a message oriented reliable multicast protocol for distributed virtual environments.

In the construction of a Distributed Virtual Reality Environment (DVE), a reliable and efficient multicast protocol on the Internet is necessary [1]. First, lets put this paper in a broader perspective by describing its relevance in the development of a highly dynamical distributed virtual environment.

One of the bottlenecks in virtual environments has always been the availability of sufficient network bandwidth to allow the participating objects to communicate with each other [2]. With the introduction of multicasting this problem was partly solved, but most traditional multicast protocols have two drawbacks [3].

The first is that these protocols are based on best effort approaches, i.e. message delivery is not guaranteed. In order to achieve this guarantee, reliable multicast protocols were introduced [4]. Although there are already many such protocols, none is optimized for distributed virtual environments [5]. Since almost all of the existing reliable multicast protocols aim to send a relatively large data chunks (e.g. a file) from one source site, they are not suitable for application in a DVE. A DVE typically has many source sites, the size of the data is relatively small and there are a large number of messages. Also, to our knowledge, an implementation of such a protocol in Java, which might a priori have some performance and timing drawbacks, has not yet been attempted.

The second problem is that multicast groups are statically allocated [6]. With virtual environments one usually considers spatial criteria to divide the world in partitions, where each partition transmits its data on one multicast group. However, 
with dynamic environments this isn't sufficient anymore. Participants have a tendency to flock together and this leads to situations where some groups are very heavily used, while others are completely idle. Allocating multicast groups in a dynamical way can solve this problem [7]. Techniques that can be used for this include probing [8] and fuzzy clustering [9]. With these methods one can determine at runtime which participants should be put together in the same multicast groups at any given moment in time.

In the classification of reliable multicast protocols $[3,12]$ the approach that we use is most closely related to the Transport Protocol for Reliable Multicast. When one classifies protocols on the basis of data buffering mechanisms [13], ours is a receiverinitiated approach, i.e. no acknowledgements of receipt (ACKs) are used. Instead, the receiver transmits a negative acknowledgement (NACK) if retransmission is needed, because there was an error in the message, because a skip in sequence numbers indicated a missing message or because a timeout has elapsed.

With this approach, two problems can arise: (1) a NACK implosion at the sender due to the detection of a missing packet by many receivers, and (2) buffer size limitations at the sender side. Indeed, in principle, the sender needs to keep all messages available for retransmission because a NACK may arrive at any time. One never knows whether all interested parties have successfully received the message. This leads to the fact that buffers should in principle be infinite.

Waiting a pseudo-random time interval before sending a NACK solves the first problem. Also, when a client is waiting to send a NACK and in the mean time it receives a NACK-request from another client for the same missing packets, it can drop his own request. The second problem is solved heuristically by assuming that messages are of no further interest after a configurable amount of time as indicated above. As indicated before, this is appropriate in VR applications.

\section{Design and Implementation}

We have implemented our reliable multicast protocol taking into account a number of design features and goals:

1. The protocol will be used in distributed virtual reality systems. From previous work [10] we know that this has some interesting implications.

- The typical message size used in virtual reality applications is rather small (< $1 \mathrm{kB})$ because once the viewers know what an object looks like and where it is positioned, one only needs to transmit the changes with respect to that information.

- Because a frame rate of $30 \mathrm{~Hz}$ is considered acceptable, there is no point in sending more than 30 update messages per second.

- When dead reckoning algorithms - i.e. determination of the current position on the basis of previous positions - are applied, an update rate of once per second will often suffice.

- When a message doesn't arrive during the first few seconds after is has been sent, it has completely lost its relevance to the virtual world. 
Based on the average message size and the maximum number of message sent per second, we can make a realistic prediction about buffer sizes and timeout windows that are key parameters in implementing the reliability in the protocol. It can also improve performance because we do not need to resize our buffers while in action. Because we know the average and the maximum throughput, we can apply the Usage Parameter Control (UPC) algorithm a.k.a. leaky bucket algorithm. This algorithm can be used to control bandwidth usage. An example is its use in ATM networks [11].

And most importantly, we can relax the reliability criteria. It is appropriate for our problem to have the sender buffer messages, for possible retransmission, only for a certain amount of time and then discard them. The amount of time may vary depending on the type of message. This way we can assign each message an importance factor. Important messages should be kept longer in the buffer.

2. The protocol has to be implemented in JAVA. The main motivation is that the virtual reality system that is being designed will be implemented in Java. We chose Java because it has features that we want to use, such as multithreading, loading classes across the network and the write-once-run-everywhere strategy. However, choosing Java as the implementation language adds an extra difficulty because it is not the optimum choice for time sensitive applications.

3. In view of the need for easy maintainability and portability, we have put significant effort into obtaining a good design. We were rigorous in defining interfaces with ease of use in mind, and made extensive use of Design Patterns [14, 15].

4. The primary design goal for our VR system is that it has to be distributed. The termination of a node, due to crash or transmission failure over an extended period, should have minimal impact on the whole. As a consequence, the reliable multicast architecture also needs to be completely distributed. This means that every participating node should be able to operate independently of all others to ensure the functioning of the protocol and in particular its reliability.

For more details on the implementation and the particular algorithms that were used, we refer to [16].

\section{Test Environment}

The experiments were performed using 45 Pentium-III computers. Each has a 450 $\mathrm{MHz}$ processor, $64 \mathrm{MB}$ memory and a 3Com 100 Mbit NIC. The network is a 100 Mbit Ethernet. The PCs are split in 5 groups, each group interconnected by a $100 \mathrm{Mb}$ HUB. A $100 \mathrm{Mb}$ switch connects these 5 groups. All computers have Windows 98 as operating system and run Sun's JDK 1.2.2

In a third paper we will compare the reliable multicast protocol between different operating systems, different computers and different Java Virtual Machines. 


\section{Benchmarks for Java-Based Multicasting}

In this paragraph we describe the artefacts we encountered while searching for the boundaries of multicasting in Java using our test environment described above.

The first thing one should wonder about when researching multicast behaviour is trying to find ways to determine how the Java API handles multicasting. The first experiment we designed had as sole purpose to check whether the Java-send() method is blocking or not. We checked this by sending a datagram every 10 milliseconds. From figure 1 one can see that the gap between the actual amount of bytes sent and the theoretical maximal amount increases with increasing datagram size. This gap signifies the time needed to actually send the datagram. And as such we can conclude that $\operatorname{send}()$ is a blocking method. If this would have been an asynchronous message, throughput per second would be proportional to the datagramsize as 10 milliseconds is more than time enough to send one datagram.

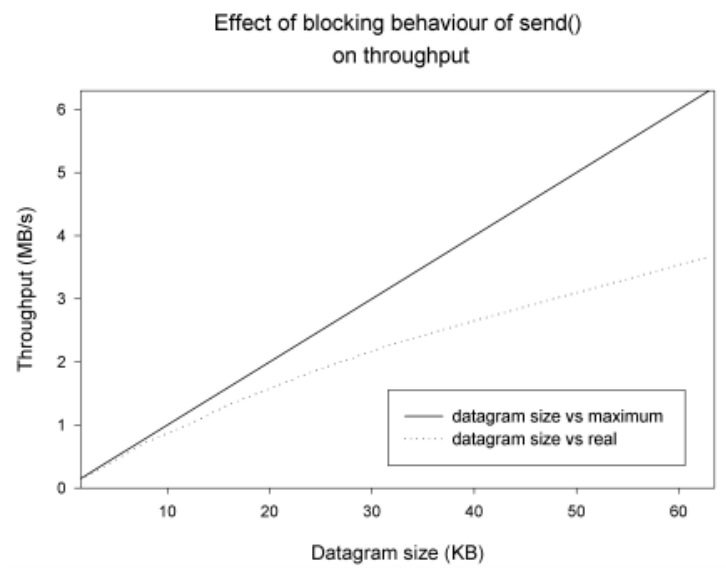

Fig. 1. The Java-send() operation is clearly a blocking operation. The larger the datagram sent, the larger the gap between the actual throughput and the maximal throughput.

In a typical VR application you have many nodes sending data to each other at the same time. In a second experiment we examined how, in our test environment, java multicast behaves when multiple servers are active at the same time. We have chosen to configure the servers as such that they each send a one-kilobyte packet every 33 milliseconds, the maximum values for VR applications. This way we can easily compare the results with those from our reliable multicast system. From figure 2 one can see that throughput is linear until we reach about 35 senders. After this, there are too many collisions and the increase in throughput flattens.

It is important to know what the ideal datagram size is. To discover this we designed the following experiment in which a server sends as many datagrams as possible of varying size. It has to be noted that multicast is build upon UDP and the maximum datagram size is 63 bytes. Figure 3 shows how throughput increases with increasing datagram size. This increase in throughput is dramatic for datagram sizes less than 8 kilobytes. After this, the increase is only average. From this graph one can 
deduct three things. First, there isn't much sense in using datagrams larger than 8 kilobytes, secondly, when the datagrams are very small, throughput drops significantly.

Effect of \#servers on total throughput

(timeout=33ms; packetsize $=1 \mathrm{~KB}$ )

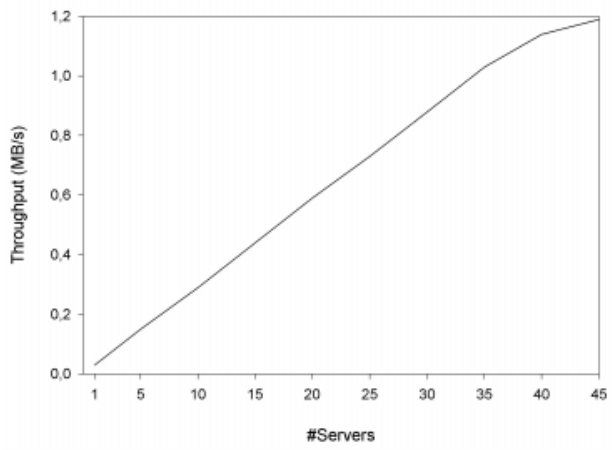

Fig. 2. As the number of servers increase, throughput increases linearly until there is a throughput of approximately $1.2 \mathrm{MB} / \mathrm{s}$. This is due to the increasing amount of collisions and to the fact that it takes about $0.9 \mathrm{~ms}$ to handle one datagram.

Since in VR application, the typical message size is less than 1 kilobyte, one can see from this graph that in a configuration with one server, it is not possible to send more than approximately $5.6 \mathrm{MB} / \mathrm{s}$. When multiple servers are active, the effect of collisions has to be taken into account (see figure 4).

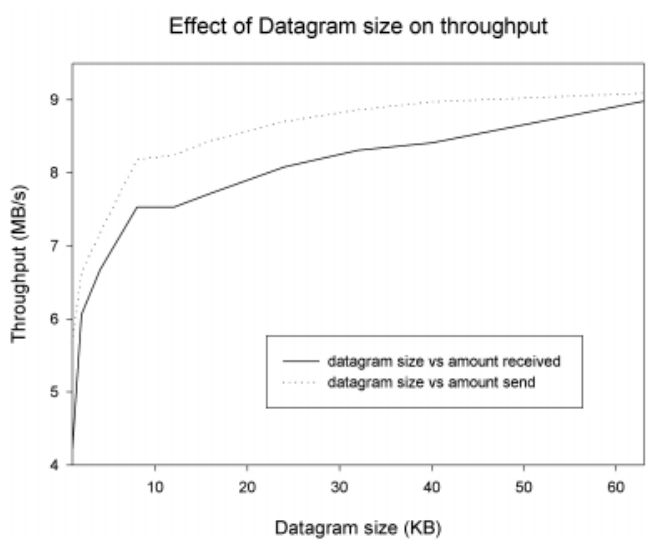

Fig. 3. For datagram sizes smaller than $8 \mathrm{~KB}$, small increases result in major throughput gains. After this, throughput increases only moderately. Also note that there are about $10 \%$ missing datagrams. This is mainly due to the NIC being unable to handle all the datagrams fast enough.

The third observation one can make is that there is a discrepancy between the amount sent and the amount received. For normal multicast this isn't a real problem, but for reliable multicast systems, this can become a problem, as all datagrams have to be received. 
The next experiment is used to determine the effect of pausing between consecutive sends. The server is configured to continuously send a $8 \mathrm{~KB}$ packet and wait a configurable amount of time.

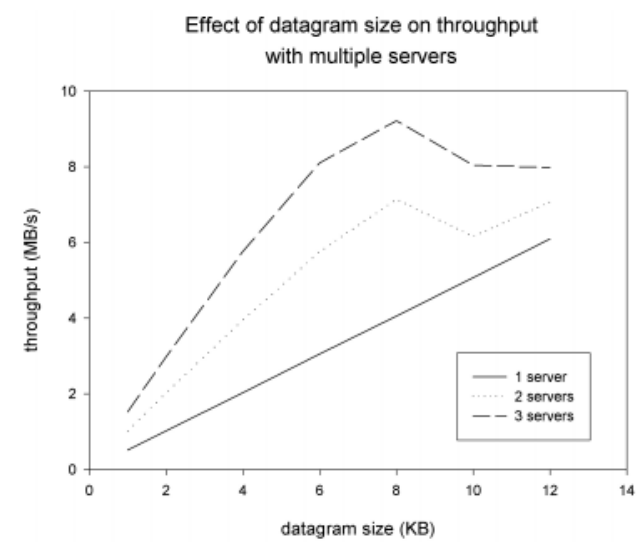

Fig. 4. When multiple servers are active, there is danger of collisions; we see a performance drop when datagrams become larger than $8 \mathrm{~KB}$.

Figure 5 shows that without waiting, a maximum throughput of $7.5 \mathrm{MB} / \mathrm{s}$ can be achieved. This leads us to the conclusion that sending a 8KB packet takes about 0.9 $\mathrm{ms}$. There the smallest amount of time we can wait is $1 \mathrm{~ms}$, the slightest pause between sends, will halve the maximum throughput, as can be seen from figure 5 . Since our multicast system uses the leaky bucket algorithm [11] to control congestion, we have to wait between consecutive sends. This implies that we won't be able to send more than about 520 messages a second. But for VR applications this is more than enough.

\section{Performance Analysis}

Since in a typical VR environment one has many participants, each sending its information, it's very important to construct an experiment that measures performance when many different servers are active. For this experiment we assumed the typical VR settings: the messages sent are one packet in size and each packet is $1 \mathrm{~KB}$. These messages are sent at a rate of 30 a second. Figure 6 shows how throughput evolves when we active more and more senders each sending $30 \mathrm{~KB} / \mathrm{sec}$. From figure 5 one can see that throughput increases until we reach 300 datagrams a second. This seems to be the maximum amount of datagrams our multicast system can handle. After this, throughput slowly degrades due to increasing datagram losses. When our VR environment needs more participating servers, we must either lower throughput, for example by using dead reckoning algorithms, or work with multiple multicast groups. An important note that has to be made is that performance and scalability is very dependant on the Operating System en Java Virtual Machine used. These observations will be discussed in an upcoming paper. Unfortunately, network conditions aren't always optimal. As such, it is important to determine the stability of 
our protocol under problematic situations. For this we created an artificial error rate by dropping a certain percentage of all datagrams just before they are to be sent. For this experiment we had 1 server sending at a rate of 30 messages a second. Each message is $1 \mathrm{~KB}$ in size.

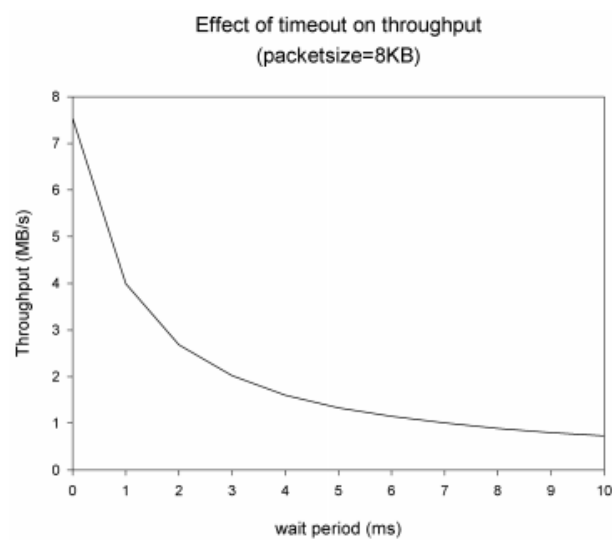

Fig. 5. When there is no pause between two consecutive sends, the application reaches its maximal throughput. This is approximately 7.6 MB. Slight pausing periods will severely lower total throughput.

From figure 7 we see that throughput remains very good, even under high error rates. This can be explained because we continuously put new messages in the system regardless whether the old ones were completely sent or not. A disadvantage of this is that the load on the servers increases with degrading networks.

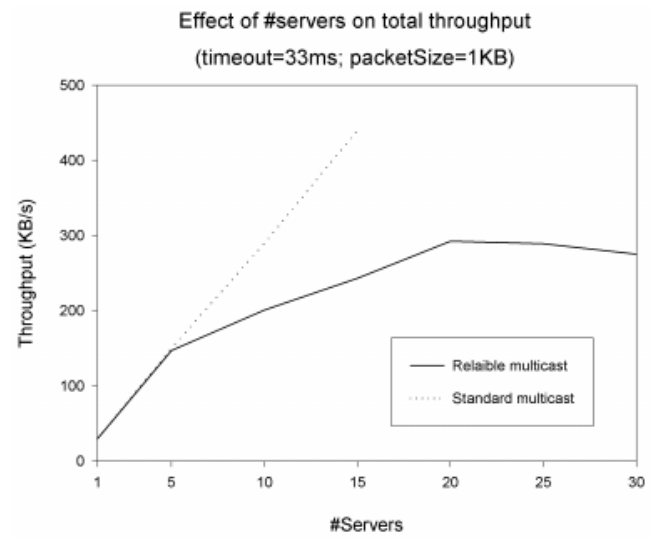

Fig. 6. Multiple servers, each sending at a rate of 30 datagrams a second, will initially increase the total throughput. At approximately $300 \mathrm{~KB} / \mathrm{s}$ there is a breakpoint, which indicates that this is the maximal throughput the Reliable Multicast System can handle.

The third test was designed to see how different message sizes would influence throughput. From figure 3 we know that an $8 \mathrm{~KB}$ message would be ideal, but a 
typical VR-message is only $1 \mathrm{~KB}$ and as such we would have a tremendous overhead. This is why we chose to fix the datagram size at $1 \mathrm{~KB}$ and test what the throughput will be when we send large messages consisting of multiple datagrams.

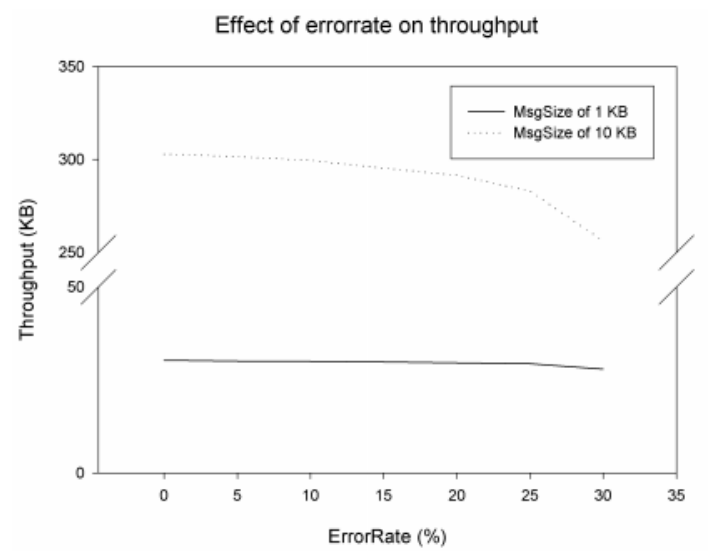

Fig. 7. For small messages, the error rate doesn't influence the total throughput because the server on sending new messages at the same rate. For large messages however, the server has to lower the injection of new messages because the maximal amount of sends per second is reached.

Once again we had a server sending at a ratio of 30 messages as second. This time however the message size varies from 1 till 80 packets, with each packet being $1 \mathrm{~KB}$. As one can see from figure 8 , there is almost no influence at all. From this we can conclude that handling large messages is as efficient as handling small ones. This test demonstrates that the used data structures work as expected.

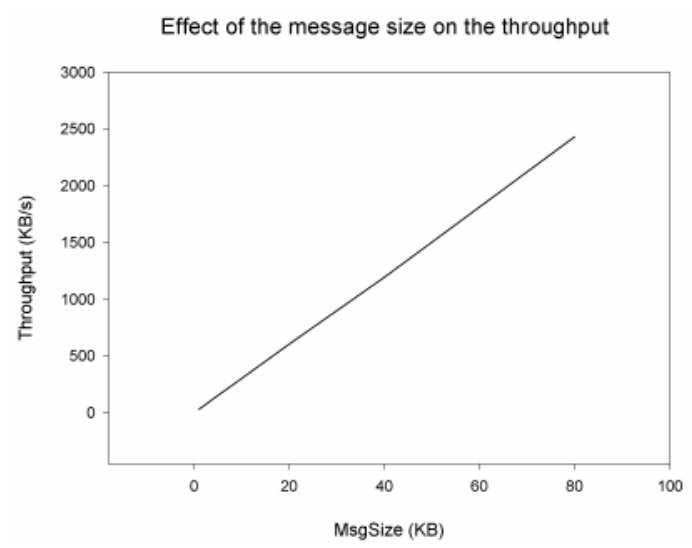

Fig. 8. Total throughput increases linearly with the message size. This indicates that the fragmentation and assembly algorithms work as expected.

The fourth and last experiment was designed to check how well our reliable multicast protocol would work when applied in other areas. For this we varied the amount of 
messages sent per second. Each message is $1 \mathrm{~KB}$ in size. As one can see from figure 9, the protocol remains efficient for large send frequencies. The flattening at the end of the curve indicates that our limit is at 400 sends a second. Which is very good when you consider that the maximum for standard, non-reliable, multicast is about 520 messages a second.

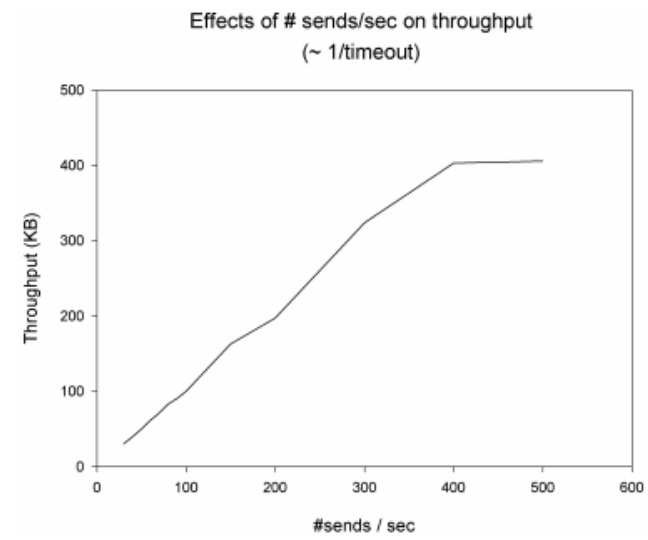

Fig. 9. As the number of sends a second increase, the throughput increases with it. From a send rate of approximately 400 messages a second there is stagnation in the throughput. This indicates that this is the maximum send rate of the Reliable Multicast System.

\section{Future Work}

This reliable multicast protocol is an important element in a much bigger project: the creation of a highly dynamical distributed virtual environment.

The next step will be minimizing the problems still remaining as stated above. The design and implementation of the probe classes [16], which will strongly decrease the total amount of messages sent when new objects enter the virtual world, will be considered in the near future. This technique is based upon the idea of sending chunks of code to the participants instead of data. This code will be able to negotiate whether the two objects are interested in each other, or not. If they are, the multicast groups on which they transmit their data will be exchanged.

As a next step, we will implement the fuzzy clustering algorithm [9] to dynamically allocate a fixed set of multicast groups to all participating objects. The most challenging task will be to define the criteria, which will determine which objects should be grouped together at a certain moment in time. To make this mechanism as flexible as possible, the criteria will be described in XML [17]. 


\section{Conclusions}

We think that we can safely conclude that the current version of the reliable multicast protocol for distributed virtual environment written in Java meets its performance targets inherent to the design goals. Due to the constantly improving performance and growing feature set of Java, the construction of a full blown distributed virtual reality system becomes more and more plausible. The protocols presented in this and future papers can only help to make the communications scheme more reliable, dynamic, performing and scalable.

\section{References}

1. Fumiaki Sato, Kunihiko Minamihata, Hisao Fukuoka, Tadanori Mizuno, "A Reliable Multicast Framework for Distributed Virtual Reality Environments", Proceedings of the 1999 International Workshop on Parallel Processing.

2. Michael J. Zyda, "Networking Large-Scale Virtual Environments", Naval Postgraduate School, Monterey, California, USA.

3. Katia Obraczka, "Multicast Transport Protocols: A survey and taxonomy", IEEE Communications Magazine, January 1998, pp. 94-102.

4. Kara Ann Hall, "The implementation and evaluation of reliable IP multicast", University of Tennessee, Knoxville, USA, 1994.

5. Kenneth P. Birman, "A Review of experiences with reliable multicast", Software Practice and Experience 29(9), 741-774 (1999)

6. Chris Greenhalgh, "Dynamic, embodied multicast groups in MASSIVE-2", Technical Report NOTTCS-TR-96-8, University of Nottingham, UK, 1996.

7. Chris Greenhalgh, "Spatial Scope and Multicast in Large Virtual Environments", Technical Report NOTTCS-TR-96-7 University of Nottingham, UK, 1996

8. Gunther Stuer, Jan Broeckhove, Frans Arickx, "A message oriented reliable multicast protocol for a distributed virtual environment”, ICSE'99 (CS-163)

9. C. Looney, "Fuzzy Clustering: A new algorithm", ICSE'99 (CS-115)

10. Kris Demuynck, Jan Broeckhove, Frans Arickx, "The VEplatform system: a system for distributed virtual reality”, Future Generation Computer Systems 14 (1998), pp. 193-198.

11. William Stallings, "Data \& Computer Communications, 6 ${ }^{\text {th }}$ edition", ISBN 0130843709 , pp 405

12. B. Sabata, M.J. Brown, B.A. Denny, "Transport Protocol for Reliable Multicast: TRM", Proc. IASTED International conference Networks, January 1996, pp. 143-145.

13. Brian Neil Levine, J.J. Garcia-Luna-Aceves, "A comparison of reliable multicast protocols", Multimedia Systems 6 (1998), pp. 334-348

14. Erich Gamma, Richard Helm, Ralph Johnson, John Vlissides, "Design Patterns", AddisonWesley.

15. Gunther Stuer, Jan Broeckhove, Frans Arickx, "Design and Implementation of a Reliable Multicast Protocol for Distributed Virtual Environments written in Java", submitted to the EuroMedia 2001 Conference.

16. Gunther Stuer, Frans Arickx, Jan Broeckhove, "A message oriented reliable multicast protocol for J.I.V.E.”, Parco99, Parallel Computing - Fundamentals \& Applications, pp. 681-688.

17. S. Laurent, "Building XML Applications", Osborn McGraw-Hill. 